\title{
Preoperative chemotherapy and corticosteroids: independent predictors of cranial surgical-site infections
}

\author{
Bryan A. Lieber, MD, ${ }^{1,2}$ Geoffrey Appelboom, MD, PhD, ${ }^{2-4}$ Blake E. Taylor, BS, ${ }^{2,3}$ \\ Franklin D. Lowy, MD, ${ }^{5}$ Eliza M. Bruce, BS, ${ }^{2,3}$ Adam M. Sonabend, MD, ${ }^{3,6}$ \\ Christopher Kellner, MD, ${ }^{2,3}$ E. Sander Connolly Jr., MD, ${ }^{2,3,6}$ and Jeffrey N. Bruce, MD,3 \\ ${ }^{1}$ Department of Neurosurgery, New York University; ${ }^{2}$ Cerebrovascular Lab, ${ }^{3}$ Department of Neurosurgery, ${ }^{4}$ The Gabriele \\ Bartoli Brain Tumor Laboratory, and ${ }^{5}$ Division of Infectious Diseases, Department of Medicine, Columbia University; and \\ ${ }^{6}$ Neuro-Intensive Care Unit, Columbia University Medical Center, New York, New York
}

\begin{abstract}
OBJECTIVE Preoperative corticosteroids and chemotherapy are frequently prescribed for patients undergoing cranial neurosurgery but may pose a risk of postoperative infection. Postoperative surgical-site infections (SSIs) have significant morbidity and mortality, dramatically increase the length and cost of hospitalization, and are a major cause of 30-day readmission. In patients undergoing cranial neurosurgery, there is a lack of data on the role of patient-specific risk factors in the development of SSIs. The authors of this study sought to determine whether chemotherapy and prolonged steroid use before surgery increase the risk of an SSI at postoperative Day 30 .
\end{abstract}

METHODS Using the national prospectively collected American College of Surgeons National Surgical Quality Improvement Program (ACS NSQIP) database for 2006-2012, the authors calculated the rates of superficial, deep-incisional, and organ-space SSIs at postoperative Day 30 for neurosurgery patients who had undergone chemotherapy or had significant steroid use within 30 days before undergoing cranial surgery. Trauma patients, patients younger than 18 years, and patients with a preoperative infection were excluded. Univariate analysis was performed for 25 variables considered risk factors for superficial and organ-space SSIs. To identify independent predictors of SSIs, the authors then conducted a multivariate analysis in which they controlled for duration of operation, wound class, white blood cell count, and other potential confounders that were significant on the univariate analysis.

RESULTS A total of 8215 patients who had undergone cranial surgery were identified. There were 158 SSIs at 30 days (frequency 1.92\%), of which 52 were superficial, 27 were deep-incisional, and 79 were organ-space infections. Preoperative chemotherapy was an independent predictor of organ-space SSIs in the multivariate model (OR $5.20,95 \% \mathrm{Cl}$ $2.33-11.62, p<0.0001)$, as was corticosteroid use (OR 1.86, 95\% Cl 1.03-3.37, $p=0.04)$, but neither was a predictor of superficial or deep-incisional SSIs. Other independent predictors of organ-space SSIs were longer duration of operation (OR 1.16), wound class of $\geq 2$ (clean-contaminated and further contaminated) (OR 3.17), and morbid obesity (body mass index $\left.\geq 40 \mathrm{~kg} / \mathrm{m}^{2}\right)(\mathrm{OR} 3.05)$. Among superficial SSIs, wound class of 3 (contaminated) (OR 6.89), operative duration (OR 1.13), and infratentorial surgical approach (OR 2.20) were predictors.

CONCLUSIONS Preoperative chemotherapy and corticosteroid use are independent predictors of organ-space SSIs, even when data are controlled for leukopenia. This indicates that the disease process in organ-space SSIs may differ from that in superficial SSIs. In effect, this study provides one of the largest analyses of risk factors for SSIs after cranial surgery. The results suggest that, in certain circumstances, modulation of preoperative chemotherapy or steroid regimens may reduce the risk of organ-space SSIs and should be considered in the preoperative care of this population. Future studies are needed to determine optimal timing and dosing of these medications.

http://thejns.org/doi/abs/10.3171/2015.4.JNS142719

KEY WORDS surgical-site infection; NSQIP; 30-day readmission; chemotherapy; steroids; predictors of infection

ABBREVIATIONS ACS NSQSIP = American College of Surgeons National Surgical Quality Improvement Program; ASA = American Society of Anesthesiologists; $B$ MI = body mass index; $C N S=$ central nervous system; $C O P D=$ chronic obstructive pulmonary disease; $C P T=$ Current Procedural Terminology; IQR = interquartile range; $\mathrm{OR}=$ odds ratio; SIRS = systemic inflammatory response syndrome; $\mathrm{SSI}=$ surgical-site infection; WBC $=$ white blood cell.

SUBMITTED December 1, 2014. ACCEPTED April 8, 2015.

INCLUDE WHEN CITING Published online November 6, 2015; DOI: 10.3171/2015.4.JNS142719. 
$\mathrm{E}$ VERY year, more than 100,000 cranial neurosurgical procedures are performed nationally (http://hcupnet. ahrq.gov). Patients are frequently prescribed regimens of corticosteroids or chemotherapy for days to weeks preoperatively, depending on the diagnosis. In neurosurgery, neoadjuvant chemotherapy often plays a role in the management of neoplasms, and steroids are very useful in decreasing cerebral edema, inflammation, and symptoms

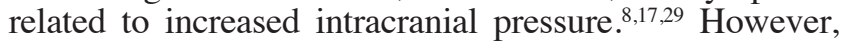
both therapies may compromise immunity; neutropenia is a well-known complication of chemotherapy, and corticosteroids broadly attenuate the action of leukocytes and inflammatory mediators. ${ }^{2-4,10,26}$ It is less established, however, whether these therapies affect the risk of surgical-site infections (SSIs) in cranial neurosurgery patients after data are controlled for potential confounders.

SSIs are among the most common types of hospitalacquired infections and are responsible for increased mortality, poorer functional outcome, 1 million days in excess hospital stay, and more than $\$ 1.5$ billion in additional health care expenditures. ${ }^{7}$ In neurosurgery, SSIs are also a major cause of early hospital readmission within 30 days of discharge..$^{5,30}$ The incidence of cranial SSIs - categorized as superficial, deep incisional, or organ spaceranges between $0.7 \%$ and $6.6 \%$ in large studies. ${ }^{9,18,32}$ Risk factors for SSIs include operative duration, advanced age, diabetes mellitus, American Society of Anesthesiologists (ASA) class, and wound class..$^{19,20,32,33}$ Knowledge of risk factors as they pertain to neurosurgery is essential in determining ideal timing for preoperative regimens, selecting optimal patients, and guiding appropriate postoperative management.

Although substantial evidence has been documented in other surgical specialties, ${ }^{16,22,34}$ less is known about the effects of chemotherapy and steroids-and patient-specific factors in general-on the risk of SSIs in cranial neurosurgery. Therapies that affect immune function may have effects on the risk of SSIs in cranial surgery that differ from those in other surgical fields..$^{21,23}$ Not only does the central nervous system (CNS) have a unique anatomical environment that includes the dura mater, it also has a distinctive immune environment with microglia and relatively few neutrophils. In the limited data that exist on the predictors of SSIs in cranial patients, it was noted in a small singlecenter study that preoperative chemotherapy trended toward significance in increasing SSI risk. ${ }^{6}$ Somewhat more data exist demonstrating the impact of corticosteroids on the risks of a broad range of postoperative infections in a wide variety of neurosurgical patients, including those who have undergone cranial, spine, or peripheral nerve procedures. ${ }^{25}$ However, there is the need for a large study that focuses on the risk of SSIs in cranial surgery and controls for factors known to specifically affect SSI risk in neurosurgical patients who undergo cranial procedures.

We hypothesized that preoperative chemotherapy and protracted steroid use increase the incidence of SSIs at postoperative Day 30. In this study, one of the largest of its kind to date, we used the American College of Surgeons National Surgical Quality Improvement Program (ACS NSQIP) database to assess the risk of chemotherapy and significant corticosteroid use on superficial and organspace cranial SSIs.

\section{Methods \\ Data Source}

The ACS NSQIP is a validated, prospectively maintained registry of information obtained from randomly assigned consenting surgical patients from several hundred academic and community hospitals nationwide. The ACS NSQIP database includes information on more than 250 variables, such as demographic information, preoperative characteristics, comorbidities, intraoperative variables, and 30-day morbidity and mortality data in both the inpatient and outpatient settings. There is a 95\% success rate in capturing outcomes and greater than $95 \%$ interrater reliability in all variables. ${ }^{31}$ Hospitals that participate in ACS NSQIP are required to employ specially trained surgical clinical reviewers, who adhere to strict variable definitions and collection methods. ${ }^{13,15} \mathrm{~A}$ variety of methods are used to collect data, including medical chart reviews, phone calls, and letters. ${ }^{31}$ Important exclusion criteria were patients younger than 18 years of age and patients treated for trauma. Hospitals are routinely audited to ensure that the data collected are standardized, and previous audits have found near-perfect agreement on the coding of most variables. ${ }^{31}$ Participating hospitals are also provided with risk-adjusted outcomes from ACS NSQIP so that they may use it as a performance measure to compare with national averages. ${ }^{15}$ Further details, including lists of inclusion and exclusion criteria in the database and wound classification criteria, are on the ACS NSQIP website (https://www.facs. org/quality-programs/acs-nsqip).

\section{Study Population and Primary Outcomes}

Prospectively collected data from the years 2006-2012 were obtained from ACS NSQIP. Cases were extracted by querying the data for cases with the primary surgeon's specialty listed as "neurological surgery." As done in previous neurosurgical studies that have queried the ACS NSQIP database,,$^{28}$ all cranial procedures were selected on the basis of ACS NSQIP-used Current Procedural Terminology (CPT) codes: 21137, 21138, 21139, 21175, 21179 21184, 21299, and 61304-62121. Patients were excluded from analysis of organ-space SSIs if they had an infection that was known preoperatively, including if they were undergoing a craniotomy or craniectomy for osteomyelitis (CPT 61501) or an abscess (CPT 61320, 61321, 61514, or 61522). Cases with a wound class of 4 or a "dirty" operative site were also excluded from our analysis of superficial SSIs. Per NSQIP criteria, wounds are classified as "clean" (Class 1), "clean-contaminated" (Class 2), "contaminated" (Class 3), or "dirty" (Class 4). Cases were also grouped into those involving transsphenoidal or ethmoidal sinus penetration (CPT 61548,61580 , or 61581 ) versus no sinus penetration, and infratentorial (CPT 61305, 61315, $61343,61345,61440,61514,61519,61520,61521$, or 61524$)$ versus supratentorial. Overall malignancy and its breakdown into primary versus secondary brain cancer were defined according to pertinent postoperative ICD-9 diagnostic codes.

The primary outcome was development of an SSI by postoperative Day 30. Infectious categories were assigned by ACS NSQIP using Centers for Disease Control guidelines. These consisted of superficial incisional infections 
(skin and subcutaneous tissue, excluding stitch abscesses), deep-incisional infections (beneath the subcutaneous tissue until the calvaria), and organ-space infections (including the calvaria down to the brain parenchyma). Only descriptive analysis was performed on deep-incisional SSIs because there was an insufficient number of this category of infections to perform a multivariate predictive analysis. Univariate analysis was performed for 25 variables considered risk factors for superficial and organ-space SSIs. These risk factors were selected on the basis of careful review of prior related SSI literature. Significant corticosteroid use was defined by ACS NSQIP as more than 10 consecutive days of oral or parenteral medication within 30 days prior to the operation. Chemotherapy, which excludes hormonal therapy, was defined as chemotherapy treatment within 30 days preoperatively. Further operational definitions for each of the perioperative risk factors are provided by the ACS NSQIP 2013 user guide (http://www.aast.org/Assets/fe526f57-5bd3-4700-94bc$497 \mathrm{~b} 035551 \mathrm{db} / 635282483441930000 /$ nsqip-definitions7-1-2013-pdf). In subsequent multivariate analysis, data were controlled for duration of operation, wound class, white blood cell (WBC) count, and other potential confounders that were significant on the univariate analysis in order to identify independent predictors of superficialincisional and organ-space SSIs.

\section{Statistical Analyses}

Analysis was performed using SPSS version 22 (IBM Corp.). Descriptive statistics are represented as the mean \pm standard deviation and interquartile range (IQR). Initially, univariate analyses were performed on patient demographic characteristics and salient preoperative variables, using the Pearson chi-square test or Fisher exact test when appropriate for categorical data and the Mann-Whitney U-test for continuous variables (e.g., total operation time). Variables with $\mathrm{p} \leq 0.20$, as well as factors with a priori clinical significance (chemotherapy, radiotherapy, WBC count, steroids, and malignancy), were entered into a multivariate logistic regression model. We used binary logistic regression with a maximum number of iterations of 20 and significance threshold of $p<0.05$. Independent predictors of SSIs were identified using this model, and their odds ratios (ORs) and 95\% CIs are presented. We employed listwise deletion for subjects missing any of the studied variables. The sample of patients with complete data for each analysis is reported. Goodness of fit for each regression model was assessed via the Hosmer and Lemeshow test, which accomplishes this by testing the null hypothesis that the difference between observed and expected events is zero. The corresponding Pearson chi-square value and $\mathrm{p}$ value were then calculated. The predictive power of our model was quantified via receiver operating characteristic (ROC) test C-statistics, which represent the area under an ROC curve and can be used to compare the goodness of fit of regression models.

\section{Results}

We identified 8215 patients from the ACS NSQIP database who met the criteria for inclusion. A total of 8098 were included in the analysis of superficial SSIs, after we excluded an additional 117 patients with a wound class of 4, since ACS NSQIP considers these patients as having a preoperative superficial infection (thus meeting our original exclusion criteria). Seventy-two patients were excluded from the organ-space SSI analysis (leaving a total of 8143) because of the presence of a preoperative abscess, which NSQIP considers a preoperative organ-space infection. Within the total population of 8215 patients, 158 patients (1.92\%) developed an SSI; 52 (0.64\%) of 8098 patients had a superficial SSI, $27(0.33 \%)$ of 8215 had a deep-incisional SSI, and $79(0.97 \%)$ of 8143 had an organ-space SSI. The mean age of our patients was 55.69 years (SD 16 years, IQR 45-67 years) and 52.4\% were female, and our ethnic distribution was consistent with the general US population (see http://www.census.gov/2010census/data/) (Table 1).

\section{Organ-Space SSI}

Of the 25 factors tested by univariate analysis (Table 2), 12 met our criteria of $p \leq 0.20$ to enter into multivariate analysis to determine independent predictors of an organspace SSI at postoperative Day 30 (Table 3). Of these 12 factors, 5 were independently significant after controlling for all other covariates (Table 3). Independent predictors of organ-space SSI were preoperative chemotherapy (OR $5.20,95 \%$ CI $2.33-11.62, \mathrm{p}<0.0001)$, chronic corticosteroid use (OR 1.86, 95\% CI 1.03-3.37, $\mathrm{p}=0.043)$, wound class ( $\mathrm{p}<0.0001$ ), prior operation within 30 days (OR 2.32, 95\% CI 1.08-5.02, $\mathrm{p}=0.03$ ), and operative duration (OR $1.16,95 \%$ CI $1.07-1.26, p<0.0001)$. Interestingly, leukopenia (defined as WBC count $<4000$ cells/ $\mu$ l) was not a statistically significant factor $(\mathrm{p}=0.75)$, but leukocytosis (defined as WBC count $>11,000$ cells/ $\mu$ l) was associated with decreased odds of SSI (OR 0.31, 95\% CI 0.15-0.64, $\mathrm{p}=0.001$ ). Patients with morbid obesity (body mass index $[\mathrm{BMI}] \geq 40 \mathrm{~kg} / \mathrm{m}^{2}$ ) also demonstrated an increased risk of infection (OR 3.05, 95\% CI 1.38-6.75, p = 0.006). A history of severe chronic obstructive pulmonary disease (COPD) strongly trended toward significance as a predictor of organ-space SSI $(p=0.06)$, but radiation therapy within 90 days of the operation was not a significant predictor $(\mathrm{p}=0.996)$. Our binary regression model demonstrated an excellent goodness of fit $\left(\chi^{2}=8.93, p=0.35\right)$ and predictability $(\mathrm{C}=0.77)$.

In a secondary analysis, malignancy was further categorized as primary brain cancer or secondary brain cancer.

TABLE 1. Demographic characteristics of the study population

\begin{tabular}{cc}
\hline Characteristic & Value \\
\hline Sex: male & $3907(47.60 \%)$ \\
\hline Age in yrs & 55.69 \\
\hline Mean & 16.21 \\
\hline SD & $45.00-67.00$ \\
\hline IQR & \\
\hline Race & $5578(78.40 \%)$ \\
\hline White & $210(3.00 \%)$ \\
\hline Asian & $770(10.80 \%)$ \\
\hline Black & $22(0.30 \%)$ \\
\hline American Indian & $524(7.40 \%)$ \\
\hline Hispanic &
\end{tabular}


TABLE 2. Univariate analysis of factors for superficial and organ-space SSIs at postoperative Day 30

\begin{tabular}{|c|c|c|c|c|c|c|}
\hline \multirow[b]{2}{*}{ Variable } & \multicolumn{3}{|c|}{ Superficial SSI } & \multicolumn{3}{|c|}{ Organ-Space SSI } \\
\hline & No. of Pts (\%) & Frequency & p Value & No. of Pts. (\%) & Frequency & $\mathrm{p}$ Value \\
\hline WBC count & & & 0.18 & & & 0.03 \\
\hline Normal & $5887(72.7 \%)$ & $0.5 \%$ & & $5917(72.7 \%)$ & $1.1 \%$ & \\
\hline$<4$ cells/ul & $270(3.33 \%)$ & $1.1 \%$ & & $272(3.34 \%)$ & $1.1 \%$ & \\
\hline$>11 \mathrm{cells} / \mu \mathrm{l}$ & $1941(24.0 \%)$ & $0.9 \%$ & & $1954(24.0 \%)$ & $0.5 \%$ & \\
\hline BMI $\left(\mathrm{kg} / \mathrm{m}^{2}\right)$ & & & 0.29 & & & 0.04 \\
\hline$<18.5$ & $509(5.59 \%)$ & $0.6 \%$ & & $518(6.36 \%)$ & $0.8 \%$ & \\
\hline $18.5-24.99$ & $2318(28.6 \%)$ & $0.6 \%$ & & $2326(28.6 \%)$ & $1.2 \%$ & \\
\hline $25-29.99$ & $2675(33.0 \%)$ & $0.6 \%$ & & $2689(33.0 \%)$ & $0.7 \%$ & \\
\hline $30-34.99$ & $1522(18.8 \%)$ & $0.7 \%$ & & $1527(18.8 \%)$ & $1.2 \%$ & \\
\hline $35-40$ & $643(7.94 \%)$ & $0.6 \%$ & & $650(7.98 \%)$ & $0.8 \%$ & \\
\hline$>40$ & $431(5.32 \%)$ & $0.9 \%$ & & $433(5.32 \%)$ & $2.3 \%$ & \\
\hline Age in yrs & & & 0.12 & & & 0.15 \\
\hline $18-40$ & $1515(18.7 \%)$ & $0.8 \%$ & & $1897(23.3 \%)$ & $1.4 \%$ & \\
\hline $41-65$ & $4185(51.7 \%)$ & $0.7 \%$ & & $4208(51.7 \%)$ & $1.0 \%$ & \\
\hline $66-79$ & $1888(23.3 \%)$ & $0.4 \%$ & & $1525(18.7 \%)$ & $0.6 \%$ & \\
\hline $80-89$ & $466(5.75 \%)$ & $0 \%$ & & $469(5.76 \%)$ & $0.6 \%$ & \\
\hline$\geq 90$ & $44(0.54 \%)$ & $1.9 \%$ & & $44(0.54 \%)$ & 0.00 & \\
\hline Current smoker & & & 0.12 & & & 0.47 \\
\hline Yes & $1767(21.8 \%)$ & $0.9 \%$ & & $1797(22.1 \%)$ & $1.1 \%$ & \\
\hline No & $6331(78.2 \%)$ & $0.6 \%$ & & $6346(77.9 \%)$ & $0.9 \%$ & \\
\hline$>2$ ETOH drinks/2 wks preop & & & 0.57 & & & 1.00 \\
\hline Yes & $340(4.20 \%)$ & $0.9 \%$ & & $346(4.25 \%)$ & $0.9 \%$ & \\
\hline No & $7758(95.8 \%)$ & $0.6 \%$ & & $7797(95.8 \%)$ & $1.0 \%$ & \\
\hline Severe COPD & & & 0.87 & & & 0.15 \\
\hline Yes & $348(4.30 \%)$ & $0.6 \%$ & & $349(4.29 \%)$ & $1.7 \%$ & \\
\hline No & $7750(95.7 \%)$ & $0.6 \%$ & & $7794(95.7 \%)$ & $0.9 \%$ & \\
\hline Disseminated cancer & & & 0.31 & & & 0.23 \\
\hline Yes & $798(9.85 \%)$ & $0.5 \%$ & & $805(9.89 \%)$ & $0.9 \%$ & \\
\hline No & $7300(90.1 \%)$ & $0.7 \%$ & & $7338(90.1 \%)$ & $1.4 \%$ & \\
\hline Malignancy & & & 0.51 & & & 0.13 \\
\hline None & $5856(72.3 \%)$ & $0.7 \%$ & & $5895(72.4 \%)$ & $0.8 \%$ & \\
\hline Primary brain & $1402(17.3 \%)$ & $0.4 \%$ & & $1403(17.2 \%)$ & $1.4 \%$ & \\
\hline Secondary & $840(10.4 \%)$ & $0.6 \%$ & & $845(10.4 \%)$ & $1.1 \%$ & \\
\hline Chronic steroids & & & 0.97 & & & 0.004 \\
\hline Yes & $921(11.4 \%)$ & $0.7 \%$ & & $916(11.2 \%)$ & $1.9 \%$ & \\
\hline No & $7177(88.6 \%)$ & $0.6 \%$ & & $7227(88.8 \%)$ & $0.9 \%$ & \\
\hline$>10 \%$ body weight loss 6 mos preop & & & 0.62 & & & 0.67 \\
\hline Yes & $147(1.82 \%)$ & $0.6 \%$ & & $153(1.88 \%)$ & $1.3 \%$ & \\
\hline No & $7951(98.2 \%)$ & $0.7 \%$ & & $7990(98.1 \%)$ & $1.0 \%$ & \\
\hline $\begin{array}{l}\text { Transfusion }>4 \text { units PRBCs } 72 \text { hrs } \\
\text { preop }\end{array}$ & & & 0.29 & & & 1.00 \\
\hline Yes & $52(0.64 \%)$ & $1.9 \%$ & & $53(0.65 \%)$ & $0.00 \%$ & \\
\hline No & $8046(99.4 \%)$ & $0.6 \%$ & & $8090(99.3 \%)$ & $1.0 \%$ & \\
\hline Intra/postop transfusion & & & 0.77 & & & 0.68 \\
\hline Yes & $520(6.42 \%)$ & $0.4 \%$ & & $537(6.59 \%)$ & $1.0 \%$ & \\
\hline No & $7578(93.6 \%)$ & $0.7 \%$ & & $7606(93.4 \%)$ & $1.1 \%$ & \\
\hline
\end{tabular}


TABLE 2. Univariate analysis of factors for superficial and organ-space SSIs at postoperative Day 30 (continued)

\begin{tabular}{|c|c|c|c|c|c|c|}
\hline \multirow[b]{2}{*}{ Variable } & \multicolumn{3}{|c|}{ Superficial SSI } & \multicolumn{3}{|c|}{ Organ-Space SSI } \\
\hline & No. of Pts & Frequency & $\mathrm{p}$ Value & No. of Pts & Frequency & $p$ Value \\
\hline Chemo $<30$ days preop & & & 0.20 & & & $<0.0001$ \\
\hline Yes & $240(2.96 \%)$ & $1.2 \%$ & & $242(2.95 \%)$ & $3.7 \%$ & \\
\hline No & $7858(97.0 \%)$ & $0.6 \%$ & & $7901(97.0 \%)$ & $0.9 \%$ & \\
\hline RT w/in 90 days preop & & & 0.20 & & & \\
\hline Yes & $129(1.58 \%)$ & $1.6 \%$ & & $131(1.61 \%)$ & $0.00 \%$ & \\
\hline No & $7969(98.4 \%)$ & $0.6 \%$ & & $8012(98.4 \%)$ & $1.0 \%$ & \\
\hline Preop sepsis & & & 0.04 & & & 0.76 \\
\hline None & $7493(92.5 \%)$ & $0.6 \%$ & & $7506(92.2 \%)$ & $1.00 \%$ & \\
\hline SIRS & $533(6.58 \%)$ & $0.9 \%$ & & $543(6.67 \%)$ & $0.70 \%$ & \\
\hline Sepsis & $53(0.65 \%)$ & $1.9 \%$ & & $69(0.85 \%)$ & $0.00 \%$ & \\
\hline Septic shock & $19(0.23 \%)$ & $5.3 \%$ & & $25(0.31 \%)$ & $0.00 \%$ & \\
\hline Prior op w/in 30 days & & & 0.36 & & & 0.06 \\
\hline Yes & $430(5.28 \%)$ & $0.9 \%$ & & $439(5.39 \%)$ & $1.8 \%$ & \\
\hline No & $7668(94.7 \%)$ & $0.6 \%$ & & $7704(94.6 \%)$ & $0.9 \%$ & \\
\hline Emergency case & & & 0.63 & & & 0.61 \\
\hline Yes & $1357(16.8 \%)$ & $0.7 \%$ & & $1398(17.2 \%)$ & $1.1 \%$ & \\
\hline No & $6741(83.2 \%)$ & $0.6 \%$ & & $6745(8.28 \%)$ & $0.9 \%$ & \\
\hline Wound class & & & 0.08 & & & 0.001 \\
\hline 1 (clean) & $7626(94.2 \%)$ & $0.6 \%$ & & $7606(93.4 \%)$ & $0.9 \%$ & \\
\hline 2 (clean-contaminated) & $388(4.79 \%)$ & $0.8 \%$ & & $387(4.75 \%)$ & $1.6 \%$ & \\
\hline 3 (contaminated) & $84(1.04 \%)$ & $3.6 \%$ & & $80(0.98 \%)$ & $5.0 \%$ & \\
\hline 4 (dirty) & $\mathrm{N} / \mathrm{A}$ & $\mathrm{N} / \mathrm{A}$ & 0.08 & $70(0.86 \%)$ & $1.4 \%$ & \\
\hline ASA class & & & & & & 0.31 \\
\hline 1 & $106(1.28 \%)$ & $1.9 \%$ & & $108(1.33 \%)$ & $0.0 \%$ & \\
\hline 2 & $2052(25.3 \%)$ & $0.3 \%$ & & $2067(25.4 \%)$ & $0.7 \%$ & \\
\hline 3 & $4445(54.9 \%)$ & $0.8 \%$ & & $4489(55.1 \%)$ & $1.1 \%$ & \\
\hline 4 & $1353(16.7 \%)$ & $0.4 \%$ & & $1385(17.0 \%)$ & $1.2 \%$ & \\
\hline 5 & $142(1.75 \%)$ & $0.1 \%$ & & $147(1.81 \%)$ & $0.0 \%$ & \\
\hline Hematocrit & & & & & & 0.39 \\
\hline Normal (35-55\%) & $6588(81.4 \%)$ & $0.6 \%$ & & $6600(81.1 \%)$ & $0.9 \%$ & \\
\hline$<35 \%$ & $1353(16.7 \%)$ & $0.7 \%$ & & $1379(16.9 \%)$ & $1.1 \%$ & \\
\hline$>55 \%$ & $157(1.94 \%)$ & $0.6 \%$ & & $164(2.01 \%)$ & 1.9 & \\
\hline Infratentorial & & & 0.02 & & & 0.34 \\
\hline Yes & $680(8.40 \%)$ & $1.3 \%$ & & $688(8.45 \%)$ & $1.3 \%$ & \\
\hline No & $7418(91.6 \%)$ & $0.6 \%$ & & $7455(91.6 \%)$ & $0.9 \%$ & \\
\hline Sphenoid sinus penetration & & & 0.52 & & & 0.12 \\
\hline Yes & $416(5.14 \%)$ & $0.2 \%$ & & $419(5.15 \%)$ & $0.2 \%$ & \\
\hline No & $7682(94.9 \%)$ & $0.7 \%$ & & $7724(94.9 \%)$ & $1.0 \%$ & \\
\hline Diabetes & & & 0.44 & & & 0.82 \\
\hline No & $7099(87.7 \%)$ & $0.7 \%$ & & $7132(87.6 \%)$ & $1.0 \%$ & \\
\hline Oral meds & $197(2.43 \%)$ & $0 \%$ & & $198(2.43 \%)$ & $0.5 \%$ & \\
\hline Non-insulin meds & $423(5.22 \%)$ & $0.2 \%$ & & $428(5.26 \%)$ & $0.9 \%$ & \\
\hline Insulin & $379(4.68 \%)$ & $0.5 \%$ & & $385(4.73 \%)$ & $1.3 \%$ & \\
\hline Dialysis & & & 1.00 & & & 0.53 \\
\hline Yes & $56(0.69 \%)$ & $0 \%$ & & $58(0.71 \%)$ & $1.8 \%$ & \\
\hline No & $8042(99.3 \%)$ & $0.6 \%$ & & $8085(99.3 \%)$ & $1.0 \%$ & \\
\hline
\end{tabular}

Chemo $=$ chemotherapy; $\mathrm{ETOH}=$ ethanol; meds $=$ medication(s); PRBCs $=$ packed red blood cells; Pts = patients; RT = radiotherapy. 
TABLE 3. Multivariate analysis of predictors of organ-space SSI

\begin{tabular}{|c|c|c|c|c|}
\hline \multirow[b]{2}{*}{ Variable } & \multirow[b]{2}{*}{ p Value } & \multirow[b]{2}{*}{ OR } & \multicolumn{2}{|c|}{$95 \% \mathrm{Cl}$ Bounds } \\
\hline & & & Lower & Upper \\
\hline Operative duration (hrs) & $<0.0001$ & 1.159 & 1.070 & 1.255 \\
\hline Length of hospitalization (days) & 0.189 & 1.006 & 0.997 & 1.015 \\
\hline History of severe COPD & 0.060 & 2.349 & 0.966 & 5.713 \\
\hline Corticosteroid use & 0.041 & 1.859 & 1.026 & 3.371 \\
\hline Chemo & $<0.0001$ & 5.201 & 2.328 & 11.620 \\
\hline Prior op w/in 30 days & 0.032 & 2.324 & 1.076 & 5.021 \\
\hline Wound Class 1 (clean) & $<0.0001$ & & & \\
\hline Wound Class 2 (clean-contaminated) & 0.012 & 3.232 & 1.288 & 8.109 \\
\hline Wound Class 3 (contaminated) & $<0.0001$ & 8.038 & 2.641 & 24.460 \\
\hline Wound Class 4 (dirty) & 0.474 & 2.095 & 0.276 & 15.886 \\
\hline Sinus penetration & 0.061 & 0.136 & 0.017 & 1.100 \\
\hline No malignancy & 0.495 & & & \\
\hline Primary brain cancer & 0.275 & 1.368 & 0.779 & 2.405 \\
\hline Secondary brain cancer & 0.903 & 0.951 & 0.425 & 2.129 \\
\hline 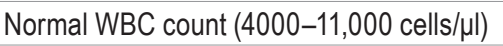 & 0.006 & & & \\
\hline WBC count $<4000$ cells $/ \mu l$ & 0.754 & 0.825 & 0.248 & 2.746 \\
\hline WBC count $>11,000$ cells/ $\mu \mathrm{l}$ & 0.001 & 0.312 & 0.152 & 0.639 \\
\hline Normal BMI (18.5-24.9 kg/m²) & 0.047 & & & \\
\hline Underweight (BMl <18.5 kg/m²) & 0.338 & 1.586 & 0.617 & 4.075 \\
\hline Overweight (BMI 25-29.9 kg/m²) & 0.897 & 0.958 & 0.503 & 1.827 \\
\hline Obese (BMI 30-34.9 kg/m²) & 0.128 & 1.660 & 0.865 & 3.188 \\
\hline Very obese (BMI 35-39.9 kg/m²) & 0.986 & 1.009 & 0.371 & 2.748 \\
\hline Morbidly obese (BMI >40 kg/m²) & 0.006 & 3.049 & 1.377 & 6.753 \\
\hline Age $16-40$ yrs & 0.307 & & & \\
\hline Age $41-65$ yrs & 0.123 & 0.655 & 0.382 & 1.122 \\
\hline Age $66-79$ yrs & 0.035 & 0.448 & 0.212 & 0.945 \\
\hline Age $80-89$ yrs & 0.420 & 0.600 & 0.174 & 2.077 \\
\hline Age $\geq 90$ yrs & 0.998 & 0.000 & 0.000 & 0.000 \\
\hline RT w/in 90 days of op & 0.996 & 0.000 & 0.000 & 0.000 \\
\hline
\end{tabular}

Neither were independent predictors of SSIs (primary: OR $1.37,95 \%$ CI $0.78-2.41, \mathrm{p}=0.28$; secondary: OR $0.95,95 \%$ CI $0.43-2.13, p=0.90$ ), and our results were unchanged (chemotherapy: $\mathrm{p}<0.0001$, OR 5.20, 95\% CI 2.33-11.62; steroids: $\mathrm{p}=0.041$, OR 1.86, 95\% CI 1.03-3.37; WBC < 4: $p=0.754$, OR $0.83,95 \%$ CI $0.25-2.75 ;$ WBC $>11: p=$ 0.001 , OR $31,95 \%$ CI $0.15-0.64)$.

\section{Superficial SSI}

Of the 25 variables that were tested in the univariate analysis (Table 2), 9 met our criteria of $p<0.20$ to enter into the multivariable analysis to determine independent predictors of a superficial SSI at postoperative Day 30 (Table 4). These were operative duration, current smoker, preoperative radiation therapy within 90 days, preoperative chemotherapy within 30 days, wound class, ASA class, infratentorial cases, age, and preoperative manifestations of systemic inflammatory response syndrome (SIRS) or sepsis.

Preoperative chemotherapy, although predictive for organ-space SSIs, was not a predictor of superficial SSIs in multivariate regression (OR 1.88, 95\% CI $0.43-8.22, \mathrm{p}$ $=0.40$ ), and neither was a protracted course of steroids ( $p=0.73$, OR $0.85,95 \%$ CI 0.34-2.14). Low, normal, or high WBC count was not a significant factor in superficial SSI $(p=0.39)$. The only independent predictors of superficial infection were operative duration (OR 1.13, 95\% CI $1.02-1.25, \mathrm{p}=0.02$ ), infratentorial case (OR 2.20, 95\% CI $1.04-4.67, \mathrm{p}=0.04)$, and wound class $(\mathrm{p}=0.01)$. Our model demonstrated good predictability $(\mathrm{C}=0.72)$ and fit $\left(\chi^{2}=4.24, p=0.84\right)$.

\section{Postinfection Outcomes}

The majority of superficial, deep-incisional, and organspace SSIs occurred after discharge $(82.4 \%, 61.5 \%$, and $74.1 \%$, respectively). Although there was no significant difference in the frequency of postoperative death between patients with and those without an organ-space SSI $6.0 \%$ vs $4.8 \%, p=0.818$, respectively), patients with an organspace SSI were far more likely to have postoperative sepsis (34.9\% vs $3.3 \%, \mathrm{p}<0.0001)$ and septic shock $(8.4 \%$ vs $1.2 \%, \mathrm{p}<0.0001)$. 
Similar to organ-space SSIs, superficial SSIs did not lead to increased fatality ( $6.0 \%$ vs $1.9 \%, \mathrm{p}=0.372)$, although both were associated with poorer outcomes. Compared with patients without superficial SSIs, patients with superficial SSIs were significantly more likely to experience sepsis $(9.6 \%$ vs $3.6 \%, p=0.021)$ and require reoperation $(28.8 \%$ vs $7.8 \%, \mathrm{p}<0.0001)$. There was a trend toward an increased frequency of septic shock $(3.8 \%$ vs $1.3 \%$, p = 0.14 ) in patients with superficial SSIs, and these patients had disproportionately more sepsis and septic shock than patients who developed organ-space SSIs.

\section{Discussion}

In this study we examined whether chemotherapy and prolonged use of steroids predict superficial or organspace SSIs. After controlling for many previously identified covariates-including WBC count, wound class, and operative duration-preoperative chemotherapy and corticosteroid use were independent predictors of organ-space SSIs, but neither were significant risk factors for superficial SSIs.

Although the impact of oral or parenteral chemotherapy on immunity - and theoretically, the ability to fight infection - may be intuitively expected, its effect on postoperative infections has not been well described, particularly in neurosurgical patients. ${ }^{6}$ In one small study, Chiang et al. ${ }^{6}$ demonstrated an increased risk of SSIs on bivariate analysis, but there were only 6 patients on chemotherapy in the study. Other studies have shown an increased risk of SSIs with locally applied carmustine wafers. ${ }^{24}$ Our study is among the largest to date demonstrating an increased risk of organ-space infections-such as meningitis-specifically among neurosurgical patients treated with preoperative or neoadjuvant chemotherapy.

Since the effects of chemotherapy on organ-space SSIs remained significant even when controlling for potentially strong confounders-including malignancy, preoperative radiotherapy, operative duration, and WBC count (Table 3)-our findings suggest that chemotherapy affects the risk of organ-space SSIs in ways that are not readily identifiable in the clinical setting. Most notably, chemotherapy was an independent predictor even in the absence of leukopenia (WBC count $<4000$ cells/ $\mu$ l). In light of standard practice guidelines, according to which patients undergoing chemotherapy are generally considered to be at a higher risk of infection if they have associated leukopenia (and specifically neutropenia) ${ }^{10}$ our results suggest that even with an otherwise normal WBC count, neurosurgical patients who receive chemotherapy before undergoing cranial surgery are still at an increased risk of serious organ-space SSIs. Unlike the results of studies outside of neurosurgery, our finding that leukopenia was not predictive of organ-space SSIs in the multivariate model suggests that standard clinical tests, such as a complete blood count, may not sufficiently assess the risk of postoperative CNS organ-space infections in patients receiving chemotherapy.

Our finding that significant preoperative corticosteroid use increases the risk of an organ-space SSI may follow the similar logic that immunocompromising therapies are associated with an increased risk of SSIs. Specifically, our study focused on more than 10 days of corticosteroid use within the 30 days immediately preceding surgery, which
TABLE 4. Multivariate analysis of predictors of superficial SSI

\begin{tabular}{|c|c|c|c|c|}
\hline \multirow[b]{2}{*}{ Variable } & \multirow[b]{2}{*}{$p$ Value } & \multirow[b]{2}{*}{ OR } & \multicolumn{2}{|c|}{$95 \% \mathrm{Cl}$ Bounds } \\
\hline & & & Lower & Upper \\
\hline Operative duration (hrs) & 0.018 & 1.130 & 1.021 & 1.251 \\
\hline Current smoker & 0.282 & 1.400 & 0.758 & 2.585 \\
\hline Chemo & 0.399 & 1.884 & 0.432 & 8.223 \\
\hline RT w/in 90 days of op & 0.379 & 2.186 & 0.383 & 12.480 \\
\hline $\begin{array}{l}\text { No sepsis, SIRS, or septic } \\
\text { shock }\end{array}$ & 0.297 & & & \\
\hline SIRS & 0.441 & 1.491 & 0.539 & 4.122 \\
\hline Sepsis & 0.418 & 2.388 & 0.290 & 19.648 \\
\hline Septic shock & 0.084 & 6.995 & 0.768 & 63.729 \\
\hline Wound Class 1 (clean) & 0.010 & & & \\
\hline $\begin{array}{l}\text { Wound Class } 2 \text { (clean-contam- } \\
\text { inated) }\end{array}$ & 0.582 & 1.397 & 0.426 & 4.584 \\
\hline Wound Class 3 (contaminated) & 0.003 & 6.893 & 1.964 & 24.196 \\
\hline ASA Class 1 & 0.064 & & & \\
\hline ASA Class 2 & 0.034 & 5.705 & 1.144 & 28.450 \\
\hline ASA Class 3 & 0.031 & 2.494 & 1.086 & 5.730 \\
\hline ASA Class 4 & 0.856 & 1.116 & 0.342 & 3.646 \\
\hline ASA Class 5 & 0.631 & 1.712 & 0.190 & 15.412 \\
\hline Infratentorial location & 0.040 & 2.202 & 1.038 & 4.672 \\
\hline Age $16-40$ yrs & 0.476 & & & \\
\hline Age $41-65$ yrs & 0.975 & 0.989 & 0.491 & 1.993 \\
\hline Age $66-79$ yrs & 0.399 & 0.664 & 0.257 & 1.719 \\
\hline Age $80-89$ yrs & 0.993 & 0.000 & 0.000 & 0.000 \\
\hline Age $\geq 90$ yrs & 0.154 & 4.739 & 0.559 & 40.171 \\
\hline 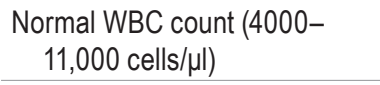 & 0.390 & & & \\
\hline WBC $<4000 \mathrm{cells} / \mu \mathrm{l}$ & 0.331 & 1.838 & 0.539 & 6.271 \\
\hline WBC $>11,000$ cells/ $\mu \mathrm{l}$ & 0.265 & 1.451 & 0.754 & 2.792 \\
\hline Corticosteroid use & 0.730 & 0.850 & 0.338 & 2.138 \\
\hline Malignancy & 0.393 & & & \\
\hline Primary brain cancer & 0.192 & 0.547 & 0.221 & 1.353 \\
\hline Secondary brain cancer & 0.503 & 0.704 & 0.252 & 1.968 \\
\hline
\end{tabular}

is generally longer than the shorter-term regimens that are frequently prescribed preoperatively for a variety of cranial procedures.$^{8,29}$ Our data suggest, in effect, that patients who are prescribed longer courses of steroids as a preoperative measure, or those who take corticosteroids for other reasons, such as rheumatologic disorders, have a higher risk of an organ-space SSI at 30 days after surgery. Our results are consistent with one of the few previous studies examining the risk of infection in neurosurgical patients on protracted corticosteroid therapy. ${ }^{25}$ In this larger study by Merkler et al., ${ }^{25}$ which broadly assessed the risks of many types of postoperative infections-including pneumonia, SSIs, and urinary-tract infections-after neurosurgical procedures, the authors found that corticosteroid use was associated with an increased risk of an organ-space SSI (OR 3.95, CI 2.29-6.79, p < 0.001). They assessed this risk in neurosurgery in a broad sense, however, with limited classification of subgroups. Indeed, organ-space SSIs are not necessarily comparable among the varying cra- 
nial, spine, and peripheral nerve surgeries. In addition, the authors did not control for certain factors, such as WBC count and duration of operation, so the effects of these potential confounders is unclear.

Merkler et al. did, however, find that while corticosteroid treatment increased the risk of organ-space SSIs in the general neurosurgical population, it did not increase the risk of superficial SSIs, a finding that is consistent with our analysis of corticosteroid use as well as for chemotherapy. In effect, these therapies appear to increase the risk of deeper SSIs largely occurring in the brain parenchyma, but not superficial SSIs of the skin and subcutaneous tissue. The underlying reason for this distinction, although unclear, may be that there are 2 separate disease processes, which not only affect different tissues but are also divided by multiple layers, including the dura, bone, and galea. In addition, the CNS has a privileged immune environment $^{14}$ that is distinct from the systemic myeloid cells in the skin and subcutaneous tissue of the scalp. For example, the CNS is relatively devoid of neutrophils and instead has microglia, ${ }^{27}$ which are largely not derived from the bone marrow $^{12}$ and have a lower turnover rate. ${ }^{11,21}$ In effect, there are a variety of potential reasons that may explain our findings that systemic chemotherapy and corticosteroids significantly impact the risk of organ-space SSIs, but not superficial SSIs, in cranial neurosurgery.

In addition to our findings regarding chemotherapy and corticosteroids, we found other independent predictors of organ-space and superficial SSIs that are consistent with previous studies, including wound class, ASA class, and operative duration. An infratentorial location was also an independent predictor of a superficial SSI, a result also consistent with previous studies. ${ }^{33}$ Interestingly, a WBC count above 11,000 cells/ $\mu$ l was associated with decreased odds of organ-space SSI. Although interpretation of this finding is limited by the nature of the database, it may be attributable to a population with a high-normal WBC count due to normal variation. Our finding that morbid obesity (BMI $\geq 40 \mathrm{mg} / \mathrm{m}^{2}$ ) is an independent predictor of organ-space SSIs has not been previously found in cranial neurosurgery, although this condition has been described as a risk factor in other surgical specialties. ${ }^{1}$ We did not find diabetes mellitus, radiation therapy within 90 days, intra- or preoperative transfusions, smoking, or advanced age ( $>65$ years) to be predictive of organ-space or superficial SSIs.

Certain limitations in our study should be noted. Although the ACS NSQIP database is a highly valuable resource that contains detailed guidelines on how to record patient characteristics and surgical outcomes, we were limited by some of the database's definitions. We could not include certain details in our analysis, including the specific type or dose of chemotherapy, corticosteroids, or radiation. We were able to categorize steroid use as "significant" because the database included a separate category for patients undergoing steroid therapy for more than 10 days. However, the database did not provide information about short-term steroid use. For chemotherapy, we were unable to assess the impact of less-common, alternative deliveries of chemotherapy. We were also unable to control for the presence or absence of preoperative antibiotics, confounding medications possibly associated with poor wound healing, or patients with acquired immunodeficiencies such as HIV infection. Other information that cannot be obtained from the database includes information about the pathogens responsible for each SSI, the WBC differential, and certain hospital characteristics (e.g., case volume, academic setting). Although surgical clinical reviewers adhere to strict ACS NSQIP variable definitions and collection methods with interrater reliability greater than $95 \%, 13,15,31$ to some degree our study may have been affected by variations in data entry or missing data. As with any large database, there are limitations to analyzing CPT codes, although we did our best to select the appropriate codes that have been used in previous ACS NSQIP studies. ${ }^{28}$ Due to the overall low incidence of SSI events in ACS NSQIP coupled with the limits of inferring clinical information from CPT codes, we did not feel that we would be able to run a reliable statistical analysis that takes into account a variety of indications for cranial neurosurgery. Also, the CPT codes for preoperative infections (e.g., abscess) are assigned immediately prior to surgery, so if an abscess were only discovered intraoperatively, it could have been included in our analysis despite actually having been present preoperatively. Although ACS NSQIP is pioneering in that it captures 30-day outcomes with minimal loss to follow-up, it does not provide us with outcomes after 30 days.

In conclusion, we report one of the first and largest analyses showing that preoperative chemotherapy and corticosteroids are independent predictors of an organ-space SSI at 30 days following cranial neurosurgery, a finding that may be related to impaired immunity in these subgroups. Our results suggest that commonly prescribed preoperative steroids and chemotherapy regimens should be optimized to provide their necessary therapeutic effect while also minimizing the risk of dangerous organ-space SSIs. Future studies may help determine the specific duration, dose, and type of chemotherapy or corticosteroids that may significantly affect the incidence of postoperative infectious complications.

\section{Acknowledgment}

The ACS NSQIP and the hospitals participating in the ACS NSQIP are the source of the data used herein; they have not verified and are not responsible for the statistical validity of the data analysis or the conclusions derived by the authors.

\section{References}

1. Angarita FA, Acuna SA, Torregrosa L, Tawil M, Escallon J, Ruíz Á: Perioperative variables associated with surgical site infection in breast cancer surgery. J Hosp Infect 79:328332, 2011

2. Barnes PJ: Anti-inflammatory actions of glucocorticoids: molecular mechanisms. Clin Sci (Lond) 94:557-572, 1998

3. Bellavance MA, Rivest S: The HPA - immune axis and the immunomodulatory actions of glucocorticoids in the brain. Front Immunol 5:136, 2014

4. Bodey GP, Buckley M, Sathe YS, Freireich EJ: Quantitative relationships between circulating leukocytes and infection in patients with acute leukemia. Ann Intern Med 64:328-340, 1966

5. Buchanan CC, Hernandez EA, Anderson JM, Dye JA, Leung M, Buxey F, et al: Analysis of 30-day readmissions among 
neurosurgical patients: surgical complication avoidance as key to quality improvement. J Neurosurg 121:170-175, 2014

6. Chiang HY, Kamath AS, Pottinger JM, Greenlee JDW, Howard MA III, Cavanaugh JE, et al: Risk factors and outcomes associated with surgical site infections after craniotomy or craniectomy. J Neurosurg 120:509-521, 2014

7. de Lissovoy G, Fraeman K, Hutchins V, Murphy D, Song D, Vaughn BB: Surgical site infection: incidence and impact on hospital utilization and treatment costs. Am J Infect Control 37:387-397, 2009

8. Dietrich J, Rao K, Pastorino S, Kesari S: Corticosteroids in brain cancer patients: benefits and pitfalls. Expert Rev Clin Pharmacol 4:233-242, 2011

9. Edwards J, Peterson K, Mu Y: National Healthcare Safety Network (NHSN) report: data summary for 2006 through 2008, issued December 2009. Am J Infect Control 37:783805,2009

10. Freifeld AG, Bow EJ, Sepkowitz KA, Boeckh MJ, Ito JI, Mullen CA, et al: Clinical practice guideline for the use of antimicrobial agents in neutropenic patients with cancer: 2010 update by the Infectious Diseases Society of America. Clin Infect Dis 52:e56-e93, 2011

11. Gehrmann J, Matsumoto Y, Kreutzberg GW: Microglia: intrinsic immuneffector cell of the brain. Brain Res Brain Res Rev 20:269-287, 1995

12. Ginhoux F, Greter M, Leboeuf M, Nandi S, See P, Gokhan S, et al: Fate mapping analysis reveals that adult microglia derive from primitive macrophages. Science 330:841-845, 2010

13. Hall BL, Richards K, Ingraham A, Ko CY: New approaches to the National Surgical Quality Improvement Program: the American College of Surgeons experience. Am J Surg 198 (5 Suppl):S56-S62, 2009

14. Heimberger AB, Sampson JH: Immunotherapy coming of age: what will it take to make it standard of care for glioblastoma? Neuro Oncol 13:3-13, 2011

15. Ingraham AM, Richards KE, Hall BL, Ko CY: Quality improvement in surgery: The American College of Surgeons National Surgical Quality Improvement Program approach. Adv Surg 44:251-267, 2010

16. Ismael H, Horst M, Farooq M, Jordon J, Patton JH, Rubinfeld IS: Adverse effects of preoperative steroid use on surgical outcomes. Am J Surg 201:305-309, 2011

17. Koehler PJ: Use of corticosteroids in neuro-oncology. Anticancer Drugs 6:19-33, 1995

18. Korinek AM: Risk factors for neurosurgical site infections after craniotomy: a prospective multicenter study of 2944 patients. Neurosurgery 41:1073-1081, 1997

19. Korinek AM, Baugnon T, Golmard JL, van Effenterre R, Coriat P, Puybasset L: Risk factors for adult nosocomial meningitis after craniotomy: role of antibiotic prophylaxis. Neurosurgery 59:126-133, 2006

20. Korinek AM, Golmard JL, Elcheick A, Bismuth R, van Effenterre R, Coriat $\mathrm{P}$, et al: Risk factors for neurosurgical site infections after craniotomy: a critical reappraisal of antibiotic prophylaxis on 4,578 patients. Br J Neurosurg 19:155-162, 2005

21. Lassmann H, Schmied M, Vass K, Hickey WF: Bone marrow derived elements and resident microglia in brain inflammation. Glia 7:19-24, 1993

22. Lawson EH, Hall BL, Ko CY: Risk factors for superficial vs deep/organ-space surgical site infections: implications for quality improvement initiatives. JAMA Surg 148:849-858, 2013

23. Lawson LJ, Perry VH, Gordon S: Turnover of resident microglia in the normal adult mouse brain. Neuroscience 48:405-415, 1992
24. McGovern PC, Lautenbach E, Brennan PJ, Lustig RA, Fishman NO: Risk factors for postcraniotomy surgical site infection after 1,3-bis (2-chloroethyl)-1-nitrosourea (Gliadel) wafer placement. Clin Infect Dis 36:759-765, 2003

25. Merkler AE, Saini V, Kamel H, Stieg PE: Preoperative steroid use and the risk of infectious complications after neurosurgery. Neurohospitalist 4:80-85, 2014

26. Nadeau S, Rivest S: Glucocorticoids play a fundamental role in protecting the brain during innate immune response. $\mathbf{J}$ Neurosci 23:5536-5544, 2003

27. Rivest S: Regulation of innate immune responses in the brain. Nat Rev Immunol 9:429-439, 2009

28. Rolston JD, Han SJ, Lau CY, Berger MS, Parsa AT: Frequency and predictors of complications in neurological surgery: national trends from 2006 to 2011. J Neurosurg 120:736745,2014

29. Ryken TC, McDermott M, Robinson PD, Ammirati M, Andrews DW, Asher AL, et al: The role of steroids in the management of brain metastases: a systematic review and evidence-based clinical practice guideline. J Neurooncol 96:103-114, 2010

30. Shah MN, Stoev IT, Sanford DE, Gao F, Santiago P, Jaques DP, et al: Are readmission rates on a neurosurgical service indicators of quality of care? J Neurosurg 119:1043-1049, 2013

31. Shiloach M, Frencher SK Jr, Steeger JE, Rowell KS, Bartzokis $\mathrm{K}$, Tomeh MG, et al: Toward robust information: data quality and inter-rater reliability in the American College of Surgeons National Surgical Quality Improvement Program. J Am Coll Surg 210:6-16, 2010

32. Valentini LG, Casali C, Chatenoud L, Chiaffarino F, UbertiFoppa C, Broggi G: Surgical site infections after elective neurosurgery: a survey of 1747 patients. Neurosurgery 62:88-96, 2008

33. Walcott BP, Neal JB, Sheth SA, Kahle KT, Eskandar EN, Coumans JV, et al: The incidence of complications in elective cranial neurosurgery associated with dural closure material. J Neurosurg 120:278-284, 2014

34. Xue DQ, Qian C, Yang L, Wang XF: Risk factors for surgical site infections after breast surgery: a systematic review and meta-analysis. Eur J Surg Oncol 38:375-381, 2012

\section{Disclosure}

Dr. Lowy reports receiving royalties from UpToDate, Inc.

\section{Author Contributions}

Conception and design: Taylor, Lieber, Appelboom. Acquisition of data: Taylor, Lieber, Appelboom. Analysis and interpretation of data: Taylor, Lieber, Appelboom, Lowy, Sonabend, Kellner, Connolly, JN Bruce. Drafting the article: Taylor, Lieber, Appelboom. Critically revising the article: Taylor, Lieber, Appelboom, Lowy, Sonabend, Kellner, Connolly, JN Bruce. Reviewed submitted version of manuscript: all authors. Approved the final version of the manuscript on behalf of all authors: Taylor. Statistical analysis: Taylor, Lieber, Appelboom. Administrative/technical/material support: EM Bruce. Study supervision: Appelboom, Connolly, JN Bruce.

\section{Correspondence}

Blake Taylor, Department of Neurosurgery, Columbia University Medical Center, 630 W. 168th St., Ste. 5-454, New York, NY 10032. email: bet2110@cumc.columbia.edu. 